NASA Technical Memorandum 106771 ICOMP-94-27

\title{
A Note on the Wave Action Den
Viscous Instability Mode on a Laminar Free-Shear Flow
}

........... Thomas F. Balsa Institute for Computational Mechanics in Propulsion Lewis Research Center Cleveland, Ohio

and University of Arizona

Tucson, Arizona

November 1994

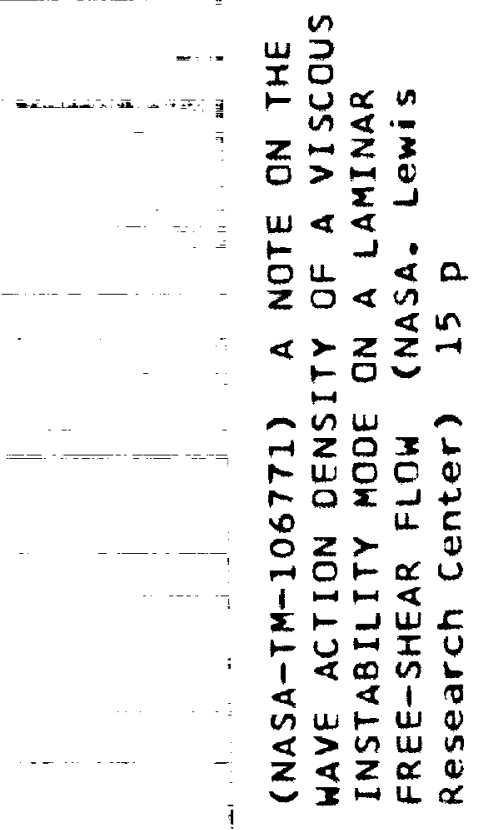

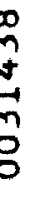

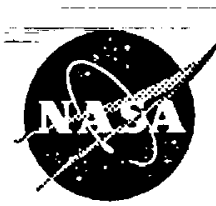

Național Aèronautics and Space Administration

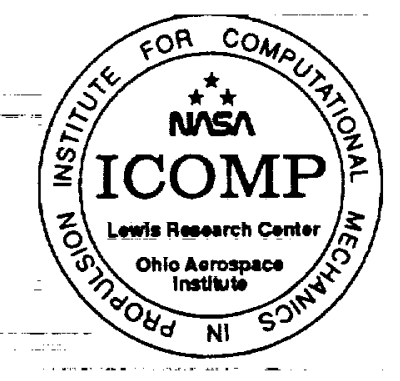


$\ldots$

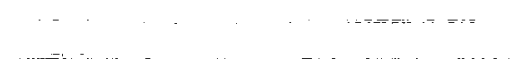

$+\cdots$

$-\quad . \quad \ldots .$.
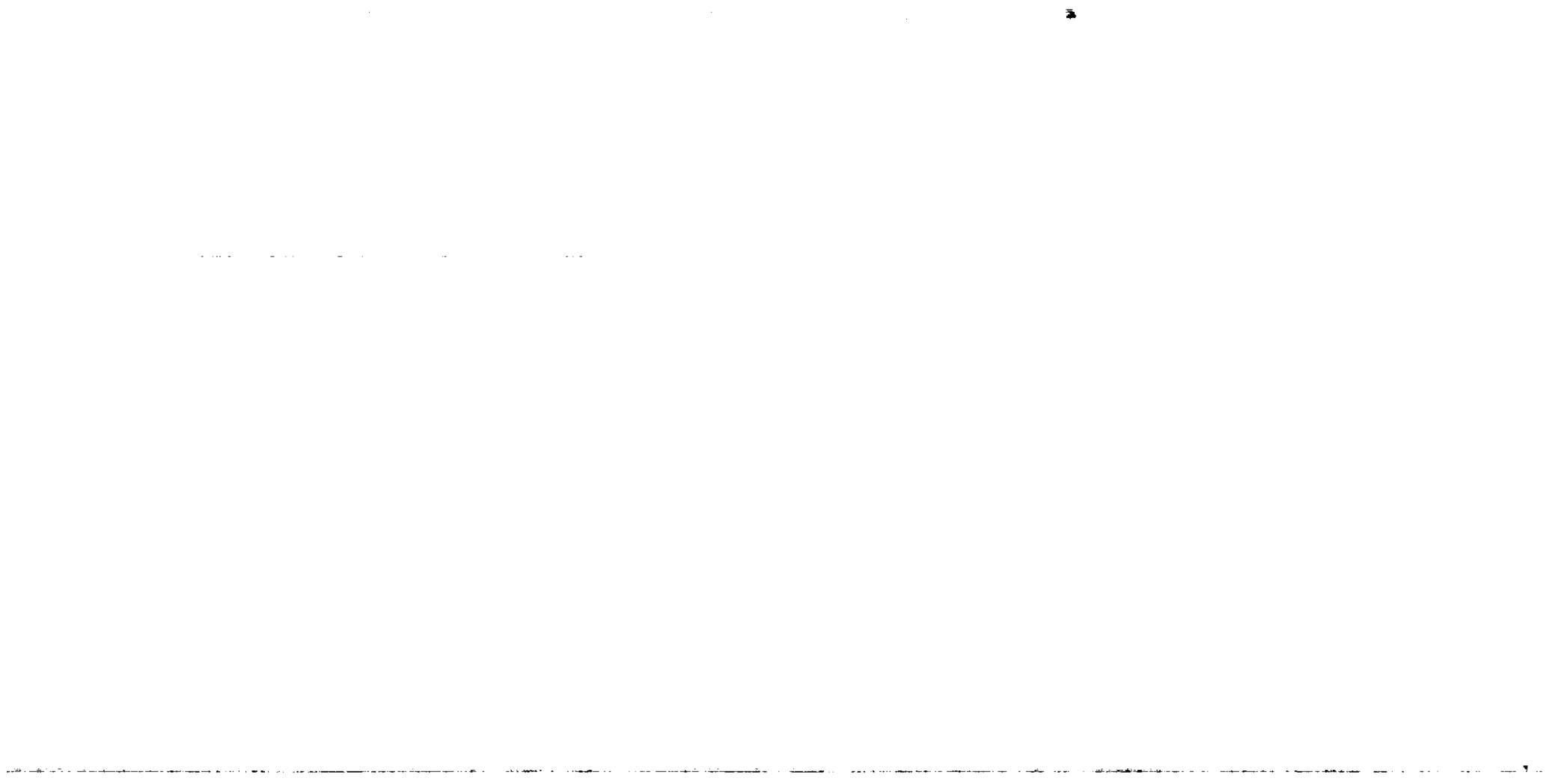

$\because-\cdots+\cdots$ 


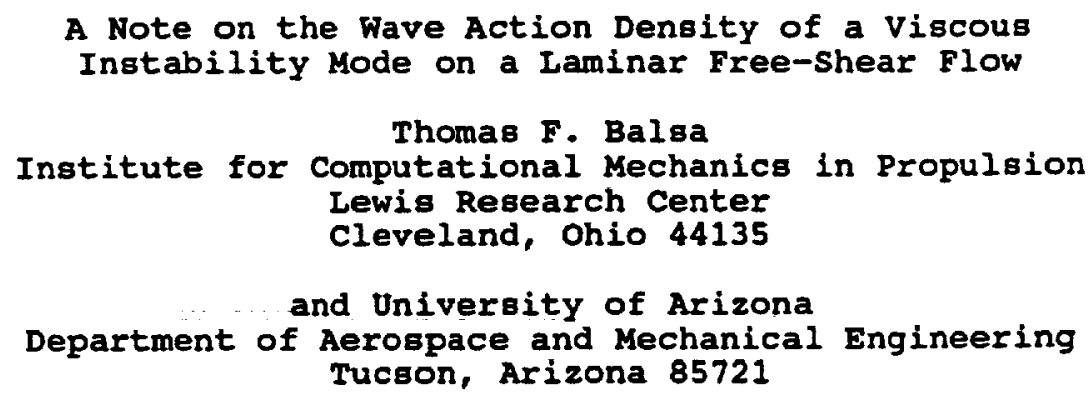

Using the assumptions of an incompressible and viscous flow at large Reynolds number. we derive the evolution equation for the wave action density of an instability wave traveling on top of a laminar free-shear flow. The instability is considered to be viscous; the purpose of the present work is to include the cumulative effect of the (locally) small viscous correction to the wave, over length and time scales on which the underlying base flow appears inhomogeneous owing to its viscous diffusion. As such. we generalize our previous work for inviscid waves. This generalization appears as an additional (but usually non-negligible) term in the equation for the wave action. The basic structure of the equation remains unaltered.

\section{Introduction}

The propagation of modal waves on slightly nonuniform or unsteady base flows can produce many interesting effects. Two important entities that describe these effects are the wave action $\mathcal{C}$ and its flux $G \mathcal{C}$, where $\mathbf{G}$ is the group velocity. In the classical theories for conservative systems, these two entities are related by a "continuity equation" that expresses a conservation law over the long spatio-temporal scales associated with the nonuniformity (or inhomogeneity) of the base flow (Whitham 1974, Hayes 1970).

Over the years, there have been many attempts to generalize these concepts to nonconservative systems, specifically, to instability waves riding on top of "diverging" base 
flows. Invariably. these generalizations are formulated in terms of an amplitude function $A$, rather than the wave action $\mathcal{A}$ (see Ho \& Huerre 1984 and references cited therein). Therefore, even in the simplest case, any correspondence between classical kinematic wave theory and the stability of non-parallel base flows is completely lost. The equation for the amplitude contains a large number of terms that are difficult to interpret. but whose combinations in a certain way form the wave action and the group velocity.

In an attempt to provide such a correspondence, the author formulated the linearized instability of non-parallel flows in terms of a complex wave action density. $\mathcal{A}$. and derived an evolution equation for this entity (Balsa 1989; also referred to as (B) herein). While the derivation in (B) is very general, it does invoke the assumption that the instability wave is inviscid; this assumption turns out to be unnecessary for a large class of flows and the analysis of (B) goes through for a viscous instability wave as well. Our purpose is to sketch out this new analysis.

In \$2, we describe the underlying base flow (essentially a free-shear layer) that is "diverging" or "non-parallel," owing to viscous diffusion. The velocity profile associated with this flow is assumed to possess an inflection point; therefore, the instability wave is nearly inviscid locally. This will be clear from the equations presented in $\$ 2$; it is the cumulative effect of the small viscous terms that we wish to capture, both for the base and perturbed flows.

In 83 , we outline the solutions for the perturbations in terms of a high-frequency ansatz. A displacement variable $\alpha$ is used in place of the velocity $\mathbf{u}$. The analysis follows that in (B) very closely; we omit most of the details and algebra for the sake of brevity. A discussion of these results can be found in $\$ 4$.

\section{Formulation of the problem}

Consider an incompressible mixing layer for the base flow with velocity $U_{B}$ and pressure $p_{B}$, respectively. The underlying Cartesian coordinate system, $x$, and the 
geometry of the problem are illustrated in figure 1. All variables are nondimensional: $\mathbf{x}=(x, y, z)$ and time, $t$, are normalized by a characteristic thickness $\left(=L_{\mathrm{ref}}\right)$ and a transit time ( $=L_{\text {ref }} / U_{\text {ref }}$ ) associated with the mixing layer, respectively. Here, $U_{\text {ref }}$ denotes a typical fluid speed (say, that in the upper external stream); this is used to nondimensionalize all fluid velocities. As is customary. pressures are measured in terms of $\rho U_{\text {ref }}^{2}(\rho=$ const. = fluid density). The relevant Reynolds number is $R e=$ $U_{\text {ref }} L_{\text {ref }} / \nu \gg 1$, where $\nu=$ const. denotes the kinematic viscosity.

The base flow may be written, to the required order of accuracy, as

$$
\begin{aligned}
& \mathrm{U}_{\mathrm{B}}=\left[1+O\left(\epsilon^{2}\right)\right] \mathrm{U}(y, \xi, \tau)+\left[1+O\left(\epsilon^{2}\right)\right] \epsilon V(y, \xi, \tau) \mathrm{e}_{2} \\
& p_{\mathrm{B}}=\text { const. }+O\left(\epsilon^{2}\right)
\end{aligned}
$$

where $\epsilon=\operatorname{Re}^{-1} \ll 1,\left(e_{1}, e_{2}, e_{3}\right)$ denote the unit vectors along the coordinate directions. and the component of $\mathbf{U}$ along the $y$-axis vanishes. The slow spatio-temporal variables, arising from the viscous diffusion of the base flow, are

$$
\xi=\epsilon\left(x e_{1}+z e_{3}\right) . \quad \tau=\epsilon t
$$

It is well known that, under the stated assumptions, the horizontal component of the base flow velocity, U. satisfies the boundary layer equations

$$
\frac{\mathrm{DU}}{\mathrm{D} t}=\epsilon \frac{\partial^{2} \mathrm{U}}{\partial y^{2}}
$$

where

$$
\frac{\mathrm{D}}{\mathrm{D} t}=\frac{\partial}{\partial t}+\mathrm{U}_{\mathrm{B}} \cdot \frac{\partial}{\partial \mathbf{x}}
$$

while the physical law of mass conservation requires

$$
\frac{\partial}{\partial \mathbf{x}} \cdot \mathbf{U}_{\mathrm{B}}=0
$$

We shall also use the definition 


$$
\mathscr{F}(\boldsymbol{y}, \boldsymbol{\xi}, \tau)=\frac{\partial^{2} \mathbf{U}}{\partial y^{2}}
$$

which (i.e., the second derivative of $\mathbf{U}$ ) plays an important role in the stability analysis.

Let us assume that this base flow is perturbed by an arbitrary disturbance whose (perturbation) velocity and pressure fields are denoted by $\mathbf{u}=\mathbf{u}(\mathbf{x}, t)$ and $p=p(\mathbf{x}, t)$. respectively. The relevant linearized equations for these quantities are

$$
\begin{array}{ll}
\text { Momentum: } & \frac{\mathrm{Du}}{\mathrm{D} t}+\mathbf{u} \cdot \frac{\partial \mathbf{U}_{\mathrm{B}}}{\partial \mathbf{x}}=-\frac{\partial p}{\partial \mathbf{x}}+\epsilon \nabla^{2} \mathbf{u} \\
\text { Continuity: } & \frac{\partial}{\partial \mathbf{x}} \cdot \mathbf{u}=0
\end{array}
$$

where $\nabla^{2}$ denotes the Laplacian operator in $(x, y, z)$ and we have assumed that the disturbances are viscous. Our interest is in the case where the perturbations are the instability waves of the base flow; quite generally. these waves are nearly inviscid when the Reynolds number is larger than about 500 (Betchov \& Szewczyk 1963) and the base-flow velocity profile possesses an inflection point.

Since the disturbance equations (3) are nondimensionalized by characteristic length and time scales that are representative of instability modes, the viscous term on the right-hand side of $(3 a)$ is indeed small. This term represents a small (local) correction to the instability modes; however, its cumulative effect over the long spatio-temporal scales, $\xi$ and $T$, is formally of order unity (Hultgren 1992). We shall incorporate this effect into the conservation equation for the wave action density, thereby generalizing the result of (B).

\section{Wave action density}

In order to obtain this generalization in the simplest way, we replace the disturbance velocity $\mathbf{u}$ by a displacement variable $\alpha=\alpha(\mathbf{x}, t)$ such that

$$
\mathbf{u}=\frac{\mathrm{D} \alpha}{\mathrm{D} t}-\boldsymbol{\alpha} \cdot \frac{\partial \mathrm{U}_{\mathrm{B}}}{\partial \mathrm{x}}
$$

A physical interpretation for $\alpha=\alpha(x, t)$ is this: Consider a fluid particle of fixed 
identity that occupies the point $\times$ (at time $t$ ) in the unperturbed base flow. The position of this same particle in the perturbed flow (at time $t$ ) is defined to be $(x+\alpha)$. In other words, the particle that would have been at $\mathbf{x}$ in the base flow is actually in a slightly different position (namely, $x+\alpha$ ) in the disturbed flow.

Using $(4)$ in $(3 a, b)$, we obtain

$$
\begin{gathered}
\frac{\mathrm{D}^{2} \boldsymbol{\alpha}}{\mathrm{D} t^{2}}=-\frac{\partial p}{\partial \mathrm{x}}+\boldsymbol{\alpha} \cdot \frac{\partial \mathcal{G}}{\partial \mathrm{x}}+\epsilon \nabla^{2} \mathbf{u} \\
\frac{\partial}{\partial \mathbf{x}} \cdot \boldsymbol{\alpha}=0
\end{gathered}
$$

where it turns out to be more convenient to leave the viscous term in its original form. The error in $(5 a)$ is of $O\left(\epsilon^{2}\right)$. It should be noted that (5) is a system of linear partial differential equations whose coefficients (i.e., $U_{\mathrm{B}}$ in $\mathrm{D} / \mathrm{D} t$ and $\mathscr{T}$ ) vary slowly with $x$, $z$, and $t$.

Clearly then, in order to obtain an asymptotic solution of (5) when $\epsilon \ll 1$, we use a high-frequency ansatz

$$
\alpha=\alpha(x, t)=\left[\alpha^{(0)}+\epsilon \alpha^{(1)}+\ldots\right] \exp (i \phi / \epsilon)+c c
$$

where $i=\sqrt{-1}$ and $\mathrm{cc}$ stands for the complex conjugate of the term immediately preceding it. Similar expansions hold for $\mathbf{u}$ and $p ; \phi=\phi(\xi, \tau)$ is the complex phase and $\alpha^{(j)}=\alpha^{(j)}(y, \xi, \tau)$ for $j=0,1$.

After substituting (6) into (5) and requiring a nontrivial solution for $(\cdot)^{(0)}$ with vanishing values at $y= \pm \infty$, we obtain the evolution equation for the phase

$$
-\frac{\partial \phi}{\partial \tau}=\Omega(\nabla \phi, \xi, \tau)
$$

where $\Omega=\Omega(\mathbf{k}, \xi, \tau)$ is, in principle, a known function of its arguments expressing the complex local frequency, $\omega=\Omega=-\partial \phi / \partial \tau$, as a function of the complex local wave vector, $\mathrm{k}=\nabla \phi$. We call $\Omega$ the dispersion relation and note that $\nabla$ denotes the gradient operator in propagation space, 


$$
\nabla=\mathbf{e}_{1} \frac{\partial}{\partial \xi}+\mathbf{e}_{3} \frac{\partial}{\partial \zeta}
$$

where $\xi=\xi e_{1}+\zeta e_{3}$. Both the frequency and the wave vector, $(\omega, k)$, are local in the sense that they depend on $\xi$ and $T$ due to the viscous spreading (and possible unsteadiness) of the base flow.

Furthermore, having satisfied the dispersion relation via $(7 a)$, the lowest-order solutions $\left(\alpha^{(0)}, p^{(0)}, u^{(0)}\right)$ must be proportional to an instability mode that can be supported by the base flow. If $p_{\mathrm{m}}=p_{\mathrm{m}}(\gamma, \xi, \tau, \mathbf{k})$ is the pressure mode at wave vector k. then we must have

$$
p^{(0)}=A(\xi, \tau) p_{\mathrm{m}}(y, \xi, \tau, \nabla \phi)
$$

where $A=A(\xi, \tau)$ is the slowly varying complex amplitude of the disturbance. Similarly.

$$
\alpha^{(0)}=A(\xi, \tau)\left(Q e_{2}-i \mathbf{W}\right), \quad \mathbf{W} \cdot \mathbf{e}_{2}=0
$$

where $(W, Q)$ represent the horizontal and vertical components of the displacement mode. They are expressible as

$$
\begin{aligned}
Q & =Q(y, \xi \cdot \tau)=\frac{1}{\omega_{0}^{2}} \frac{\partial p_{\mathrm{m}}}{\partial y} \\
\mathbf{W} & =\mathbf{W}(y, \xi, \tau)=-\frac{\nabla \phi}{\omega_{0}^{2}} p_{\mathrm{m}}
\end{aligned}
$$

and $p_{\mathrm{m}}$ on the right-hand sides of $(8 c, d)$ is to be evaluated at $\mathbf{k}=\nabla \phi$. The Dopplershifted frequency, $\omega_{0}$, is given by

$$
\omega_{0}=\omega_{0}(y, \xi, \tau)=-\left(\frac{\partial \phi}{\partial \tau}+\mathbf{U} \cdot \nabla \phi\right)
$$

The algebra is omitted for brevity; these results are discussed in more detail in (B). At this stage of the analysis, the amplitude, $A=A(\xi, \tau)$, is an arbitrary function of its arguments. 
In order to obtain the evolution equation for the wave action, we must proceed to the next order in $\epsilon$. Here, $\alpha^{(1)}$ and $p^{(1)}$ will obey linear inhomogeneous equations; the forcing terms in these equations arise from the variation of the lowest-order solutions on the long spatio-temporal scales, $\xi$ and $\tau$. In addition, the viscous term in $(5 a)$ will also contribute to the forcing in the present analysis; this is the only new term that was not already discussed in (B).

Briefly, at $O(\epsilon)$, the governing equations for $\alpha^{(1)}$ and $p^{(1)}$ are

$$
\begin{gathered}
-\omega_{0}^{2} \alpha^{(1)}+i \nabla \phi p^{(1)}+e_{2} \frac{\partial p^{(1)}}{\partial y}=\text { terms in }(\mathrm{B})+\mathbf{R} \\
i \nabla \phi \cdot \alpha^{(1)}+\mathrm{e}_{2} \cdot \frac{\partial \alpha^{(1)}}{\partial y}=\text { terms in }(\mathrm{B})
\end{gathered}
$$

where $\mathbf{R}$ represents the new term that arises from the viscous correction in $(5 a)$. It is convenient to separate this term into its vertical and horizontal components via

$$
\mathbf{R}=\mathbf{e}_{2} R_{\perp}+i \mathbf{R}_{\| 1} \quad \mathbf{e}_{2} \cdot \mathbf{R}_{\|}=0
$$

Employing (4) and (8), we find

$$
\begin{gathered}
R_{\perp}=-i A\left[\frac{\partial^{2}}{\partial y^{2}}-\nabla \phi \cdot \nabla \phi\right]\left(\omega_{0} Q\right) \\
i \mathbf{R}_{||}=-A\left[\frac{\partial^{2}}{\partial y^{2}}-\nabla \phi \cdot \nabla \phi\right]\left[\omega_{0} \mathbf{W}+Q \frac{\partial \mathrm{U}}{\partial y}\right]
\end{gathered}
$$

The notation is as follows: The expression "terms in (B)" stands for those forcing terms that have been given already by Balsa (1989); we shall not repeat them here because they are lengthy and their contribution to the evolution equation for the wave action has been calculated in (B).

Observe that

$$
\mathscr{L}=\frac{\partial^{2}}{\partial y^{2}}-\nabla \phi \cdot \nabla \phi
$$

is a representation of the Laplacian operator within the high-frequency ansatz; within 
this same representation, $\omega_{0} Q$ and $\omega_{0} \mathbf{W}$ are suitable time derivatives of the fluid displacements in cross and propagation spaces. These derivatives provide the lowestorder velocity components in these spaces (essentially $\left.u^{(0)}\right)$. with the exception that the vertical displacement, $Q$, of a material volume in the nonuniform horizontal base flow, $\mathbf{U}=\mathbf{U}(y)$, also produces a perturbation velocity in propagation space in proportion to $Q(\partial \mathrm{U} / \partial y)$. These effects are clearly contained in (10).

It is well known that the $(\cdot)^{(1)}$ solutions exist if the forcing terms in $(9 a, b)$ satisfy a solvability condition. When this condition is applied to the "terms in (B)," we obtain the result for the wave action density as presented in equation (35) of (B). This equation, in our current notation (note that $\phi$ of (B) is written as i $\phi$ herein), becomes

$$
\frac{\partial \mathcal{L}}{\partial \tau}+\nabla \cdot(\mathbf{G} \mathcal{A})+A^{2} \int_{-\infty}^{+\infty} Q \frac{\partial \mathcal{F}}{\partial y} \cdot \mathbf{W} d y=0 \quad \text { (12, also (35) in B) }
$$

where $\mathcal{A}$ and $\mathrm{G}$ are defined in $(14 b, d)$. Roughly speaking. (12) contains the effects of inviscid instability modes interacting with slightly inhomogeneous base flows.

On the other hand, when the solvability condition is applied to all the terms on the right-hand sides of $(9 a, b)$, we obtain the equation for the wave action density as presented below (14). This new equation, the main result of this paper, describes the effects of slightly viscous instability modes interacting with inhomogeneous base flows. Since the solvability condition is linear in the forcing terms, the new equation for the wave action contains all the terms presented in (12) plus a new term arising from inclusion of $\mathbf{R}$ in this condition. This is tantamount to adding the integral (see (31) of (B))

$$
-i A \int_{-\infty}^{+\infty}\left(Q R_{\perp}-\mathbf{W} \cdot \mathbf{R}_{\|}\right) d y
$$

to the left-hand side of (12). After some algebra, the final result may be written as

$$
\frac{\partial \mathcal{L}}{\partial \tau}+\nabla \cdot(\mathbf{G} \mathcal{C})+H \mathcal{K}=0
$$


where

$$
\begin{array}{r}
\mathcal{L}=\mathcal{L}(\xi, \tau)=A^{2} \int_{-\infty}^{+\infty} \omega_{0}\left(Q^{2}+\mathbf{W} \cdot \mathbf{W}\right) d y \\
H=H(\xi . \tau)=\frac{\nabla \phi \cdot \int_{-\infty}^{+\infty} \mathscr{F}\left(Q^{2}+\mathbf{W} \cdot \mathbf{W}\right) d y}{\int_{-\infty}^{+\infty} \omega_{0}\left(Q^{2}+\mathbf{W} \cdot \mathbf{W}\right) d y}
\end{array}
$$

and

$$
\mathbf{G}=\left.\frac{\partial \Omega(\mathbf{k}, \xi, \tau)}{\partial \mathbf{k}}\right|_{\mathbf{k}-\nabla \phi}
$$

We recognize $\mathbf{G}$ as the (complex) group velocity. The complex function $H$ represents the interaction between the (viscous) wave and the non-uniform base flow; in the classical theories, $H=0$, thereby reducing $(14 a)$ to the familiar conservation equation for the wave action, $\mathcal{K}$ (but not for the amplitude $A !)$.

We emphasize that the integral in (13) contributes only to the interaction function $H$. Also, there are many equivalent ways of writing this contribution (as well as $H$ itself; see §4). In order to cast (13) into a simple and useful form, it is necessary to recall that $\left(\omega_{0} Q\right)$ satisfies Rayleigh's equation for the vertical velocity, the pressure mode, $p_{\mathrm{m}}$, satisfies Rayleigh's equation for the pressure, and $Q$ and $\mathbf{W}$ are expressible in terms of $p_{\mathrm{m}}$ via $(8 c, d)$. The accompanying algebra is reasonably straightforward but, perhaps, not entirely trivial.

\section{Discussion and conclusions}

In summary, (14a) provides the evolution equation for the complex wave action density, $\mathcal{A}$, of viscous instability waves interacting with slightly inhomogeneous base flows arising from the viscous diffusion of the latter. The complex interaction function, $H$, contains additively both the cumulative effects of the inhomogeneity and those of the 
viscous correction to the wave. As such, it is the most general result that can be obtained within the framework of small-disturbance theory. Note that $(14 a)$ is to be solved "simultaneously" with the equation for the phase, $(7 a)$, in order to provide $\partial \phi / \partial \tau$ and $\nabla \phi$ in the former (though. strictly speaking. these equations are decoupled in the linearized theory).

It is instructive to recast the numerator of $(14 c)$ in a form that separates the effects of the base flow inhomogeneity from those of the viscous correction for the waves. After using some of the tricks mentioned at the end of $\$ 3$ and performing a couple of integrations by parts, we find

$$
\nabla \phi \cdot \int_{-\infty}^{+\infty} \mathscr{F}\left(Q^{2}+\mathbf{W} \cdot \mathbf{W}\right) d y=\int_{-\infty}^{+\infty} Q \frac{\partial \mathscr{F}}{\partial y} \cdot \mathbf{W} d y-2 \int_{-\infty}^{+\infty} \frac{1}{\omega_{0}} \frac{\partial \omega_{0}}{\partial y} Q \mathscr{F} \cdot \mathbf{W} d \mathbf{y}
$$

where the first term on the right-hand side of (15) is in complete agreement with the "inviscid" result in (12). Clearly, the second term is entirely responsible for the viscous correction to the instability waves.

Unfortunately, calculation of the terms in (15) can be done only numerically: it entails solving the boundary layer equations (2) for U, solving the inviscid Rayleigh equation for the dispersion relation $\Omega=\Omega(\mathbf{k}, \xi, \tau)$ and for the displacement modes $Q$ and $W$, obtaining the phase $\phi=\phi(\xi, \tau)$ via $(7 a)$, and then evaluating the integrals in (15) numerically. This is beyond the scope of the present work.

Nevertheless, it is possible to analytically calculate the terms in (15), as well as those in (14c), for a very special case. In order to do this, we think of $H=H(\xi, \tau)$ as a function (say, $\mathscr{K}$ ) in the extended space $(\mathbf{k}, \xi, \tau)$, where

$$
\mathscr{K}=\mathscr{K}(\mathbf{k}, \xi, \tau) \cdot \frac{\mathbf{k} \cdot \int_{-\infty}^{+\infty} \mathcal{F}\left(Q^{2}+\mathbf{W} \cdot \mathbf{W}\right) d y}{\int_{-\infty}^{+\infty} \Omega_{0}\left(Q^{2}+\mathbf{W} \cdot \mathbf{W}\right) d y}
$$

and the inviscid displacement modes, $Q$ and $\mathbf{W}$, are to be obtained at wave vector $\mathbf{k}$. 
In addition,

$$
\Omega_{0}=\Omega-\mathbf{k} \cdot \mathbf{U}
$$

so that

$$
H=H(\xi . \tau)=\mathscr{K}(\nabla \phi . \xi . \tau)
$$

with the understanding that $\partial \phi / \partial \tau=-\Omega(\nabla \phi, \xi, \tau)$. Similar remarks hold for (15).

The special case alluded to previously occurs when, at the point and time of interest (which define $\xi$ and $\tau$ ),

$$
\mathbf{U}=(\tanh y) \mathbf{e}_{1}
$$

and the local mode is precisely neutral: $\mathbf{k} \cdot \mathbf{k}=1$ (Michalke 1964). In this case, we have closed-form solutions for the modes and the (singular) integrals given above may be evaluated analytically by a well-known contour deformation in the complex $y$-plane. The result is

$$
\mathscr{K}=0
$$

Evidently, the contributions to $\mathscr{K}$ arising from the effects of the base flow inhomogeneity and those of the viscous correction to the instability mode (as separated out in (15)) cancel in this special case; these contributions are $\mp 8$, respectively. This suggests that there may be a (partial) cancellation arising from these two effects, even in the general case. Very roughly, we expect the inhomogeneity of the base flow to be responsible for an increase in the wave action and the viscous correction to the instability to produce a decrease in $\mathcal{N}$.

The author is grateful for the support provided by the Institute for Computational Mechanics in Propulsion (ICOMP) and the hospitality provided by the NASA Lewis Research Center during a summer (1994) visit. Discussions with Dr. L. S. Hultgren were extremely helpful in generalizing the author's previous work. 


\section{REFERENCES}

BALSA, T. F. 1989 Amplitude equations for wave packets in slightly inhomogeneous unstable flows. J. Fluid Mech. 204, 433-455.

BETCHOV, R. \& SZEwCZYK, A. 1963 Stability of a shear layer between parallel streams. Phys. Fluids 6, 1391-1396.

HAYEs, W. D. 1970 Conservation of action and modal wave action. Proc. $R$. Soc. Lond. A 320, 187-208.

Ho, C.-M. \& HUERRE, P. 1984 Perturbed free shear layers. Ann. Rev. Fluid Mech. 16. $365-424$

HULTGREN, L. S. 1992 Nonlinear spatial equilibration of an externally excited instability wave in a free shear layer. $J$. Fluid Mech. 236, 635-664.

MichAlKE, A. 1964 On the inviscid instability of the hyperbolic-tangent velocity profile. J. Fluid Mech. 19, 543-556.

WhithAM, G. B. 1974 Linear and Nonlinear Waves. Wiley. 


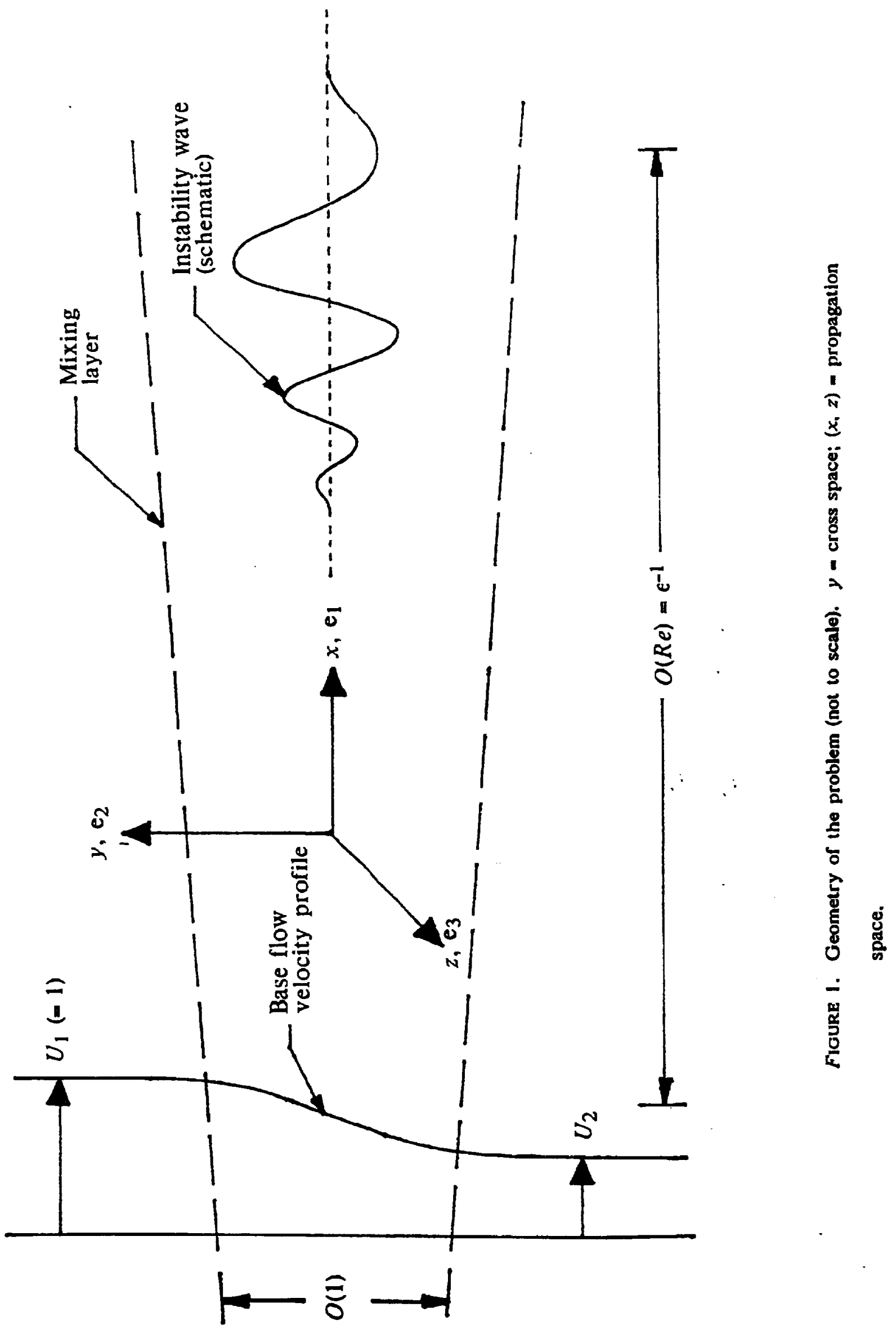




\begin{tabular}{|c|c|c|c|}
\hline \multicolumn{3}{|c|}{ REPORT DOCUMENTATION PAGE } & $\begin{array}{l}\text { Form Approved } \\
\text { OMB No. 0704-0188 }\end{array}$ \\
\hline \multicolumn{4}{|c|}{ 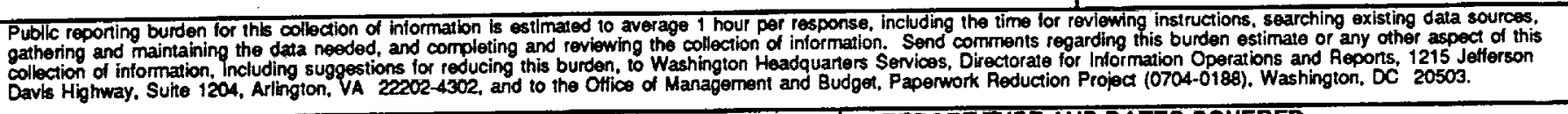 } \\
\hline 1. AGENCY USE ONLY (Leave blank) & \begin{tabular}{|l|l|}
$\begin{array}{l}\text { 2. REPORT DATE } \\
\text { November } 1994\end{array}$ \\
\end{tabular} & \multicolumn{2}{|c|}{$\begin{array}{l}\text { 3. REPORT TYPE AND DATES COVERED } \\
\text { Technical Memorandum }\end{array}$} \\
\hline \multicolumn{3}{|c|}{$\begin{array}{l}\text { 4. TITLE AND SUBTrLE } \\
\text { A Note on the Wave Action Density of a Viscous Instability Mode on a } \\
\text { Laminar Free-Shear Flow }\end{array}$} & 5. FUNDING NUMBERS \\
\hline \multicolumn{3}{|l|}{$\begin{array}{l}\text { 6. AUTHOR(S) } \\
\text { Thomas F. Balsa }\end{array}$} & $-505-90-5 K$ \\
\hline \multicolumn{3}{|c|}{$\begin{array}{l}\text { National Aeronautics and Space Administration } \\
\text { Lewis Research Center } \\
\text { Cleveland, Ohio } 44135-3191\end{array}$} & E-9219 \\
\hline \multicolumn{2}{|c|}{$\begin{array}{l}\text { National Aeronautics and Space Administration } \\
\text { Washington, D.C. } 20546-0001\end{array}$} & $\begin{array}{l}\text { 10. } s \\
\text { s } \\
1 \\
1\end{array}$ & $\begin{array}{l}\text { NASA TM-106771 } \\
\text { ICOMP-94-27 }\end{array}$ \\
\hline \multicolumn{4}{|c|}{$\begin{array}{l}\text { 11. SUPPLEMENTARY NOTES } \\
\text { Thomas F. Balsa, Institute for Computational Mechanics in Propulsion, NASA Lewis Research Center (work funded under NASA } \\
\text { Cooperative Agreement NCC3-233), and University of Arizona, Department of Aerospace and Mechanical Engineering, Tucson, } \\
\text { Arizona 85721. ICOMP Program Director, Louis A. Povinelli, organization code 2600, (216) 433-5818. }\end{array}$} \\
\hline \multicolumn{3}{|c|}{ 12a. DISTRIBUTIONAVAILABILTY STATEMENT } & TRIBUTION CODE \\
\hline \multicolumn{4}{|c|}{$\begin{array}{l}\text { Using the assumptions of an incompressible and viscous flow at large Reynolds number, we derive the evolution equation } \\
\text { for the wave action density of an instability wave traveling on top of a laminar free-shear flow. The instability is consid- } \\
\text { ered to be viscous; the purpose of the present work is to include the cumulative effect of the (locally) small viscous } \\
\text { correction to the wave, over length and time scales on which the underlying base flow appears inhomogeneous owing to } \\
\text { its viscous diffusion. As such, we generalize our previous work for inviscid waves. This generalization appears as an } \\
\text { additional (but usually non-negligible) term in the equation for the wave action. The basic structure of the equation } \\
\text { remains unaltered. }\end{array}$} \\
\hline 14. SUBJECT TERMS & & & $\begin{array}{c}\text { 15. NUMBER OF PAGES } \\
15\end{array}$ \\
\hline Instability waves; Wave ac & tion density & & $\begin{array}{r}\text { 16. PRICE CODE } \\
\mathrm{A03} \\
\end{array}$ \\
\hline $\begin{array}{l}\text { 17. SECURTY CLASSIFICATION } \\
\text { OF REPORT } \\
\text { Unclassified }\end{array}$ & $\begin{array}{l}\text { 18. SECURITY CLASSIFICATION } \\
\text { OF THIS PAGE } \\
\text { Unclassified }\end{array}$ & $\begin{array}{l}\text { 19. SECUURTY CLASSIFICATION } \\
\text { OF ABSTRACT } \\
\text { Unclassified }\end{array}$ & 20. LIMTTATION OF ABSTRACT \\
\hline NSN 7540-01-280-5500 & & & $\begin{array}{l}\text { landard Form } 298 \text { (Rev. 2-89) } \\
\text { escrbed by ANSI Std. Z39-18 }\end{array}$ \\
\hline
\end{tabular}

